\title{
Giant Pulmonary Hydatid Cyst and Trauma in a 9 Year-Old Child: A Case Report
}

\author{
Mohsen Sokouti ${ }^{1}$, Behrooz Shokouhi ${ }^{2}$, Massoud Sokouti ${ }^{3}$ and Babak Sokouti ${ }^{*}, 4$

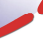 \\ ${ }^{I}$ Department of Cardiothoracic Surgery, Imam Reza Hospital, Tabriz University of Medical Sciences, Tabriz, Iran \\ ${ }^{2}$ Department of Pathology, Imam Reza Hospital, Tabriz University of Medical Sciences, Tabriz, Iran \\ ${ }^{3}$ Nuclear Medicine Research Center, Mashhad University of Medical Sciences, Mashhad, Iran \\ ${ }^{4}$ Biotechnology Research Center, Tabriz University of Medical Sciences, Tabriz, Iran
}

\begin{abstract}
Herein, we report a case of giant lung hydatid cyst in a nine-year-old boy. For four years, he experienced mild chest pain and chronic nonproductive cough. He had a trauma resulted from a fall two days before admission. Chest X-ray showed misdiagnosed massive pleural effusion, and was aspirated in the other hospital. However, after admission, the computed tomography revealed a giant lung hydatid cyst filling the right hemithorax completely. Being considered by the diagnosis of ruptured lung hydatid cyst, he was treated surgically by right-thoracotomy. The existing hydatid cyst, (e.g., with a dimension of $30 * 22 * 20 \mathrm{~cm}$ ) filled all cavity of hemithorax extended from the right diaphragm to the apex of the lung situated in the right lower lobe. After evacuation of the hydatid fluid and laminated membrane, right lower lobectomy was carried out due to remaining no salvageable parenchyma without any complications. Also, the pathologic examination have confirmed hydatid cyst. In conclusion, giant hydatid cysts are probably misdiagnosed with massive pleural effusion in the endemic area. And, because of the risk of allergic reactions, anaphylactic shock and dissemination, it should not be aspirated.
\end{abstract}

Keywords: Children, giant, hydatid cyst, lung, pleural effusion.

\section{INTRODUCTION}

Hydatid disease is an important health issue in the World Health Organization's (WHO) Eastern Mediterranean region countries [1]. Echinococcosis Gronulosis is the most common type of Echinococcosis in our endemic area. Patients' populations are usually being located in impoverished parts of the world. The life cycle of the Echinococcosis tapeworm involves canine as the definitive host, livestock and accidently humans as the intermediate hosts. After ingestion of the ova by gastrointestinal enzymes, embryos were released. Embryos hatch the mucosa and travel through the portal vessels or lymphatic and loge in the liver, lung and other organs. Pulmonary parenchyma involves the second common organ (lungs) after liver infection (10-30\%). Due to the delay presentation of symptoms of pulmonary hydatid cyst, it can grow even in giant sizes [1-3].

We propose a giant pulmonary hydatid cyst diagnosed during the trauma processing treated successfully by surgery which was misdiagnosed with pleural effusion.

\section{CASE PRESENTATION}

A nine-year old boy was admitted to our referral hospital in August 2013 with a history of trauma caused by a fall two

*Address correspondence to this author at the Biotechnology Research Center, Tabriz University of Medical Sciences, Tabriz, Iran;

Tel: +98(41)33364038; Fax: +98(41)33379420; E mail: b.sokouti@gmail.com days before admission. He had chest pain, and mild respiratory distress and four years with non productive cough. He was sent to our center due to suspicious diagnosis of ruptured giant pulmonary hydatid cysts confirmed by $5 \mathrm{ml}$ hydatid fluid thoracentesis. Chest roentgenogram, demonstrated a mass density of right hemithorax supposed to be massive pleural effusion with mediastinal shifting to the left (Fig. 1). On admission, the patient status included the mild respiratory distress (i.e., $\mathrm{RR}=29, \mathrm{PR}=80, \mathrm{~T}=37.5$ ), and

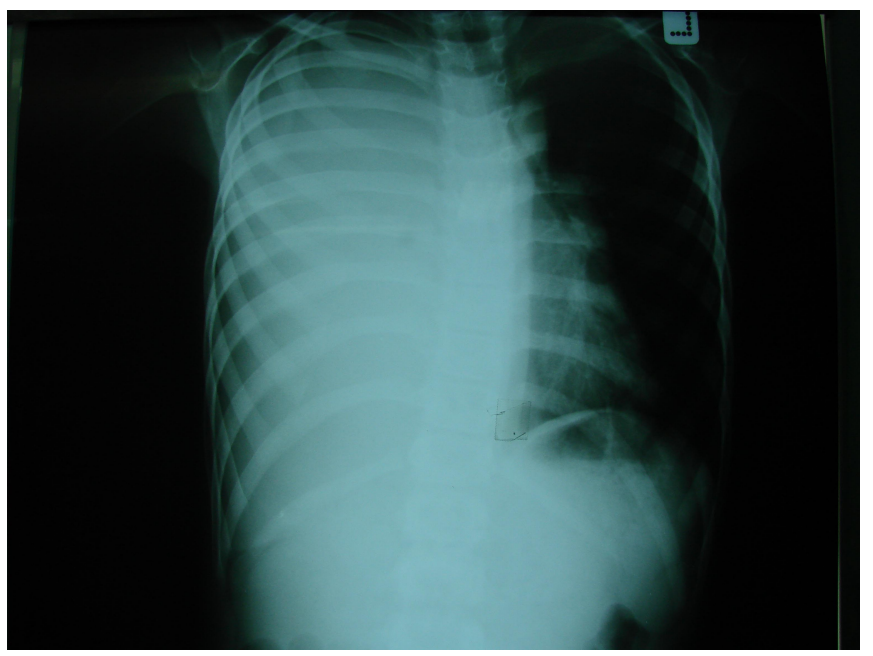

Fig. (1). The Chest X-ray shows the right hemithorax opacity shifting the mediastinum toward the left. 


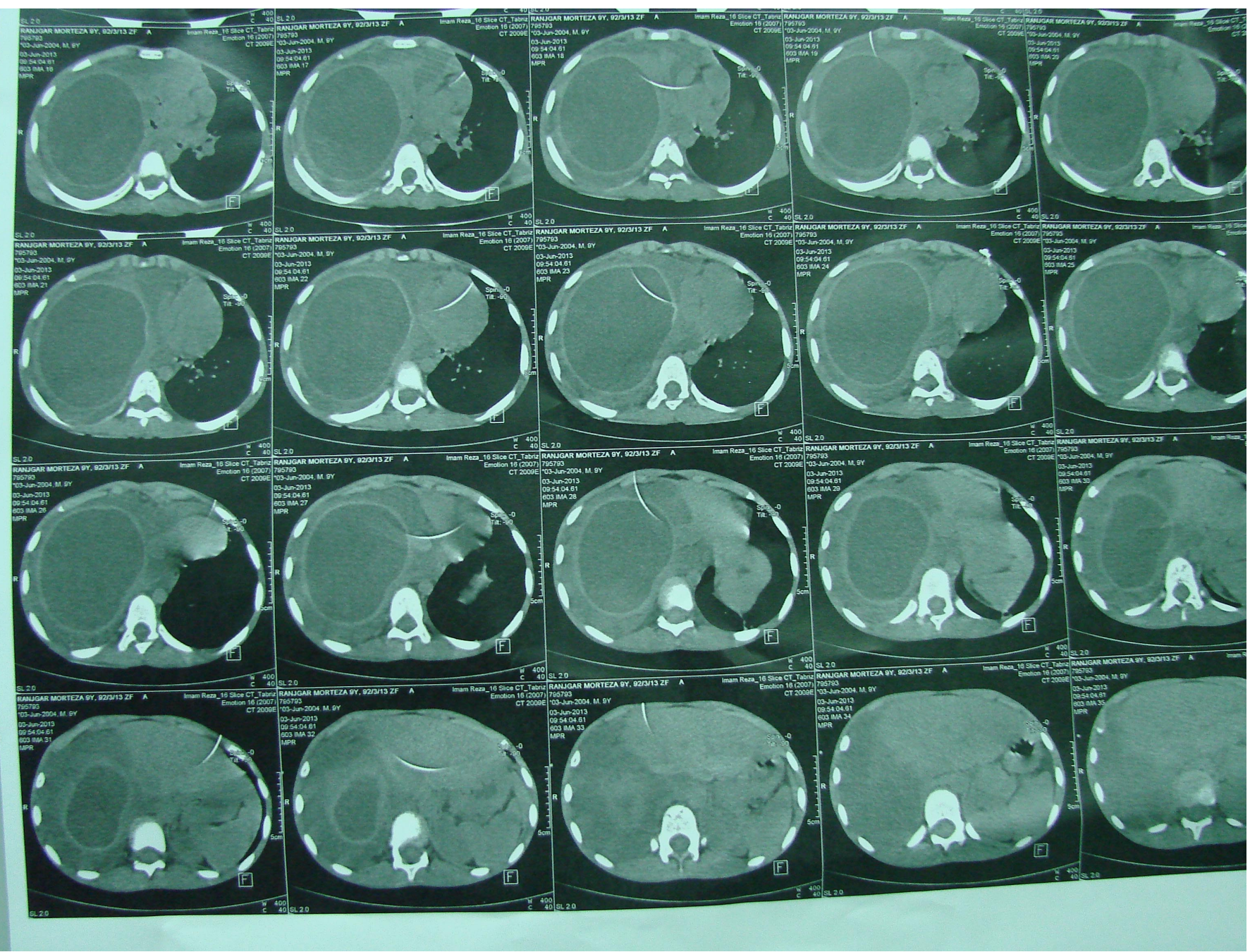

Fig. (2). Computed tomography of the chest shows right giant hydatid cyst which has filled the left hemithorax.

normal vital signs on physical examinations. Respiratory sounds were not audible over the right side lung. Serologic hemagglutination test (ELISA) for Echinococcosis was also positive. Computed tomography (CT) scan of the chest confirmed the right side pulmonary giant mediastinal cyst with mediastinal shift to the left and an absent shadow of collapsed right lung (Fig. 2). He was scheduled for perfoming the thoracotomy. After right-thoracotomy, a giant hydatid cyst with dimensions of $30 * 22 * 20 \mathrm{~cm}$ was found extending from the right diaphragm toward the apex of the lung. There was not any pneumothorax, or pleural effusion at the time of operation owing to severe adhesions of the right pulmonary hydatid cyst to the extensive surface of the chest wall. After lyses of the adhesions, the chest cavity, all around the cyst was covered with spongy hypertonic normal saline. Then, the cyst was removed without contamination, the pericyst was opened and the laminated membrane was extracted from the right lower lobe (RLL). No pulmonary parenchyma remained in the RLL, and right lower lobectomy was successfully performed. The remaining lobes of the lung were expanded completely. Two chest tubes were inserted into the hemithorax cavity, and then the chest was closed. The patient discharged the hospital on the fifth day without any complications. After six months, the controlled chest X-ray was normal (Fig. 3). He was in good condition during the six months follow up period while medically treated by Albendazole $800 \mathrm{mg} /$ day. Moreover, pathologic examinations have confirmed the existence of a pulmonary hydatid cyst (Fig. 4).

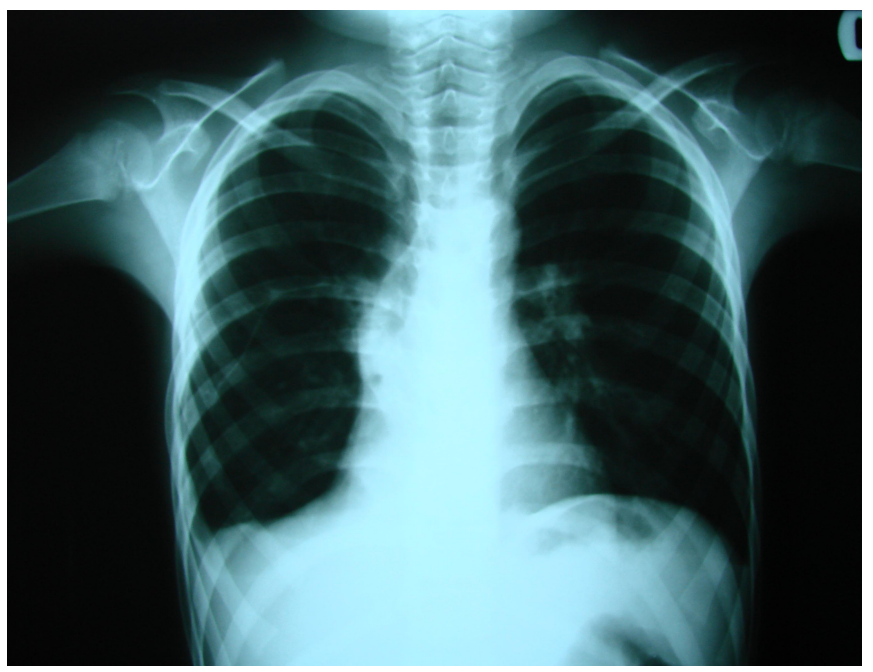

Fig. (3). Controlled chest X-ray is normal. 


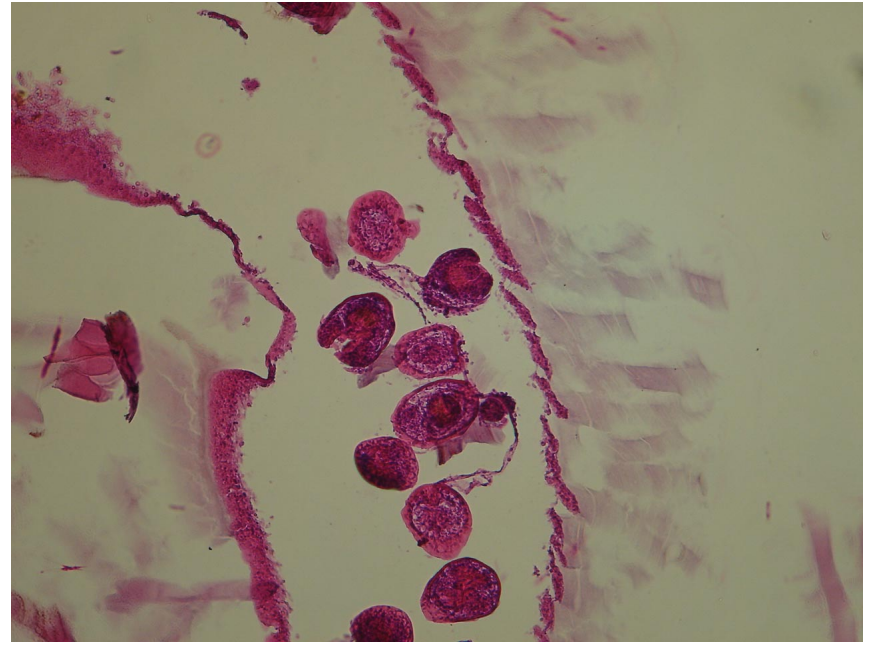

Fig. (4). Microscopic pathologic examination shows germinative layer, scolices and laminated membrane.

\section{DISCUSSION}

Pulmonary hydatid cyst may grow faster in the children's lung parenchyma than in the liver and can become very large and even giant sizes which is usually asymptomatic during growth of the cyst. This phenomenon can explain a delay in the diagnosis and treatment $[4,5]$. Higher elasticity of the lung parenchyma in children and adolescent lets this rapid growth of the cyst. There is no standard size to define the "giant" cyst and is generally accepted as being greater than $10 \mathrm{~cm}$ in diameter. In reviewing literature, giant hydatid cysts are frequently encountered in children more than ten years old. Usually, it has been shown to be symptomatic in complicated and uncomplicated cysts $[2,6]$. Cough with or without purulent sputum, chest pain, hemoptysis, chest deformity, dyspnea, nausea and vomiting can be the signs of giant pulmonary hydatid cysts. These symptoms are caused by the effect of tissue pressure caused by considerable volumes of cyst's fluid and atelectasis of the lung $[7,8]$.

Chest imaging can show the giant hydatid cyst as an opacity occupying a part or complete space of hemithorax as reported for this patient. Air fluid or water lily, meniscus or double meniscus signs can be radiological signs of complicated cysts. Based on the above mentioned imaging signs, the diagnosis of the disease can be carried out with high accuracy [9].

Computed tomography can diagnose it in $100 \%$ of patients and can exclude pleural encysted effusions, lung abscess and tumors [10]. However, chest X-ray could not diagnose the giant hydatid cyst in this patient due to falling off a height reported from the previous hospital and misdiagnosed by traumatic massive pleural effusion.
Surgery is the choice of treatment and there is no doubt that destroyed parenchyma must be resected. Although, lung parenchyma preserving procedures must be remained in mind, however, in giant sizes most of lung parenchyma are destroyed and when severe hemorrhage or suppuration of cyst cavity and destruction of more than $60 \%$ of the lobe, and no salvageable lung parenchyma are presented, segmentectomy or lobectomy are indicated $[2,4,6]$.

In conclusion, giant hydatid cysts can be probably misdiagnosed with trauma phenomenon, and it should be part of the differential diagnosis of massive pleural effusion in the endemic area. Cyst puncture should be avoided, because of the risk of allergic reactions including anaphylactic shock and possible dissemination,

\section{CONFLICT OF INTEREST}

The authors confirm that this article content has no conflict of interest.

\section{ACKNOWLEDGEMENTS}

Declared none.

\section{REFERENCES}

[1] Mahmodlou R, Sepehrvand N, Nasiri M. Saucerization: a modified uncapitonnage method of surgery for pulmonary hydatidosis. World J Surg 2013; 37(9): 2129-33.

[2] Arroud M, Afifi A, El Ghazi K, Nejjari C. Bouabdollah Y. Lung hydatid cysts in children: comparison study between giant and nongiant cysts. Pediatr Surg Int 2009; 25: 37040.

[3] Sokouti M, Pezeshkian M, Ghabili K, Gholzari EJS. Surgical procedures and postoperative complication with giant and nongiant pulmonary hydatid cysts. Life Sci J 2013; 10(1): 138-42.

[4] Harlaftis NN, Aletras HA, Symba PN. Hydatid disease of the lung In: Shields TW, Lo Cicero J, Reed CE, Feins RH, Eds. Philadelphia: General Thoracic Surgery. Lippincot: Williams \&Wilkins 2009; pp. 1187-95.

[5] Montazeri V, Sokouti M, Rashidi MR. Comparison of pulmonary hydatid disease between children and adults. Tanaffos J 2007; 6: $13-8$.

[6] Usuluer O, Ceylan KC, Kayan S, Sevi S, Gursoy S. Surgical management of pulmonary hydatid cysts. Is size an important prognostic indicator? Thex Heart Inst J 2010; 37:429- 34

[7] Topcu S, Kurul IC, Tastepe I. Bozkurt D, Gulhan E, Cetin G. Surgical treatment of pulmonary hydatid cyst in children. J Thorac Cardiovasc 2000; 120: 1097-101.

[8] Karaoglanoglou N, Kurkcuoglu C, Goryuner M, Eroglu A, Turkyilmaz A. Giant hydatid cysts. Eur J Cardiothorac Surg 2001; 19: 914-7.

[9] Sokouti M, Sokouti B, Sokouti M. The value of X-ray and CT scan in diagnosing pulmonary hydatid cyst. Clin Imaging 2013; 37: 978.

[10] Khalilzadeh S, Baghaei N, Ghafarzadeh B, Shadmehr MB, Velayati AA. Hydatid pulmonary disease in children: a nine - year study. Tanaffos J 2004; 12: 43-8. 